\title{
Papilloedema, a complication of progressive diaphyseal dysplasia: a series of three case reports
}

Mark Wright, Neil R Miller, Robert M McFadzean, Paul Riordan-Eva, Andrew G Lee, Michael D Sanders, Gawn G Mcllwaine

\begin{abstract}
Backgroundlaims-Progressive diaphyseal dysplasia (PDD) is a rare, autosomal dominant, osteosclerotic dysplasia affecting both endochondrally and intramembranously derived bones. Severely affected patients can develop progressive stenosis of the optic canals and compressive optic neuropathy. Although raised intracranial pressure (ICP) has been described in patients with PDD in whom visual loss has occurred, the elevation of ICP in those patients has been thought to be either non-contributory or only partially responsible for the accompanying visual loss.

Methods-Three cases were reviewed and the clinical and radiological characteristics are described here.

Results-All three patients had bilateral optic disc swelling with no radiological evidence of either compressive optic neuropathy or thrombosis of the intracranial venous sinuses. The aetiology of the disc swelling was proved to be papilloedema in the first two cases and was probably the dominant cause in the third case.

Conclusion-The visual loss documented in at least two of the three patients reported appears to be solely attributable to raised ICP. Normalisation of the ICP has led to an improvement and stabilisation of the visual function in all three patients. Patients with PDD probably require periodic ophthalmic assessments. (Br F Ophthalmol 1998;82:1042-1048)
\end{abstract}

Progressive diaphyseal dysplasia (PDD) was first described in the 1920 s separately by Camurati ${ }^{1}$ and Engelmann. ${ }^{2}$ To our knowledge fewer than 100 cases have been reported worldwide. The condition is characterised by the progressive symmetrical formation of abnormal bone along both the periosteal and endosteal surfaces of the long tubular bones. ${ }^{3}$ While expressivity is variable, ${ }^{4-6}$ the cranium and axial skeleton may be involved in severely affected individuals. ${ }^{7}$ Age at onset of symptoms varies from 1 year 8 months to 70 years.

The majority of patients $(75 \%)$ present in childhood with painful limbs, an abnormal gait, and excessive fatiguability. ${ }^{7}$ These children have palpably thickened long bones and secondary muscle atrophy. Exophthalmos is present in approximately $50 \%$ of cases. ${ }^{78}$ Skull base sclerosis is present in severely affected cases with involvement of the whole skull in the most severely affected individuals. Moderate to severe involvement of the anterior cranial fossa is present in all patients exhibiting exophthalmos which is secondary to the encroachment of sclerotic bone on the orbit. ${ }^{8}$

While the underlying abnormality is unknown, PDD is thought to result from an imbalance between the normally occurring processes of bone resorption and formation that take place during skeletal maturation and following injury. Biopsy specimens of the diaphyseal region of affected long bones have shown immature woven bone that has failed to remodel into normal Haversian bone. ${ }^{59}$ An excessively high osteoblast/osteoclast ratio and a high osteoid calcium to phosphorus ratio have been observed in biopsy specimens from affected individuals. ${ }^{10}$ Ophthalmic manifestations that have previously been described ${ }^{112}$ are shown in Table 1.

\section{Methods}

Three cases of PDD were reviewed in detail.

\section{CASE 1}

This 35 year old female first presented in childhood with painful limbs and radiological changes affecting the long bones which were characteristic of PDD. Her bone pain was controlled on prednisolone $11 \mathrm{mg}$ alternate days. She presented to the National Hospital for Neurology and Neurosurgery in London in 1988 with the recent onset of headache and bilateral visual obscurations.

On examination, she was of short stature with fixed flexional deformities of the elbow and knee joints with marked bowing of the long bones, the tibias being the worst affected. In addition to appearing cushingoid, she demonstrated the typical facial characteristics seen in severely affected patients (Fig 1). Visual acuities were $6 / 6$ and N5 bilaterally with normal colour vision (Ishihara plates) and full visual fields (kinetic perimetry). Eye movements were full, exophthalmometry measured $22 \mathrm{~mm}$ on the right side and $20 \mathrm{~mm}$ on the left. Both optic discs were swollen and spontaneous venous pulsation was absent.

Table 1 Ophthalmic manifestations associated with progressive diaphyseal dysplasia

Exophthalmos

Spontaneous subluxation of the globe

Secondary hypertelorism

Disc swelling/optic atrophy

Epiphora

Lagophthalmos

Blepharoptosis

Lateral rectus palsy

Convergence insufficiency

Secondary glaucoma

Cataract 

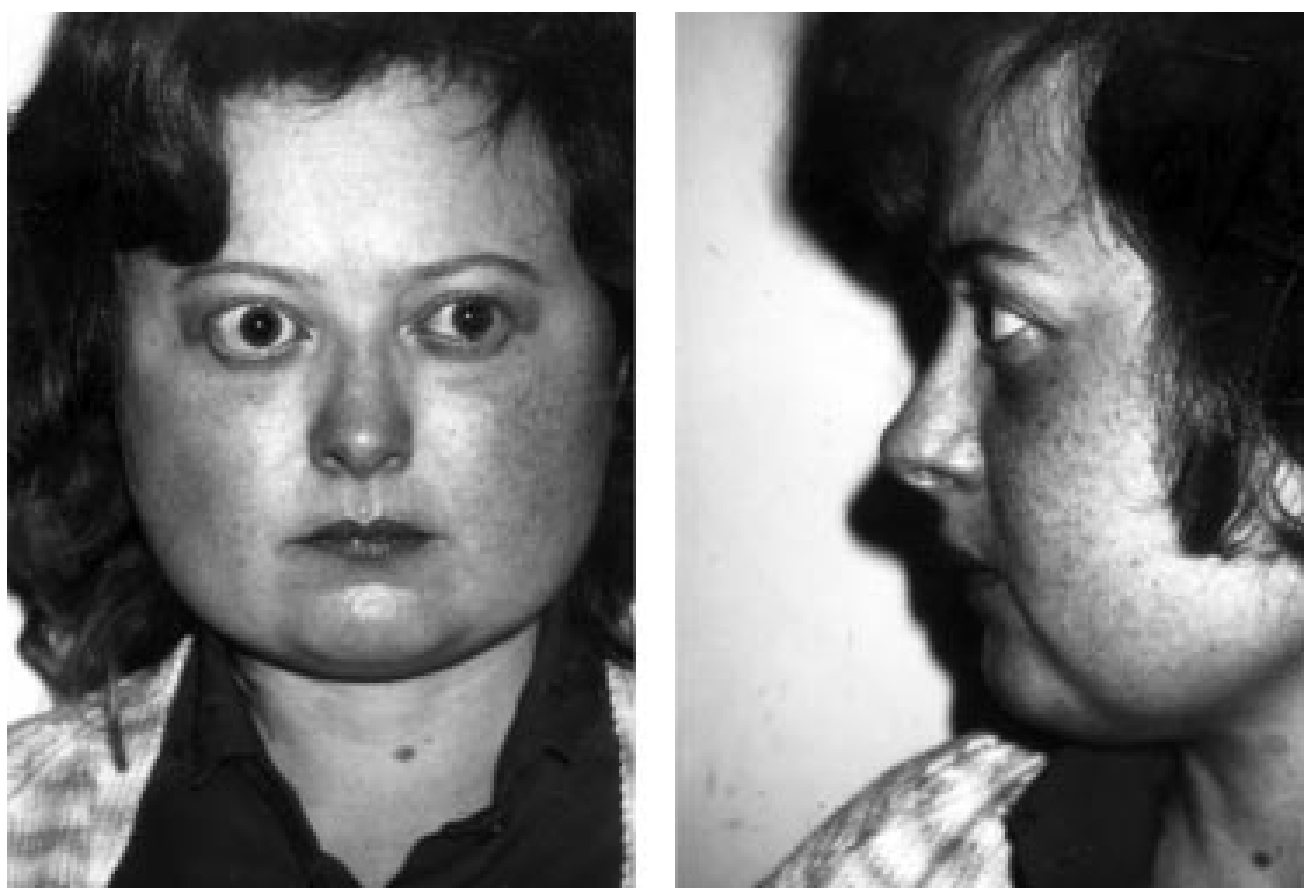

Figure 1 (A) and (B) Facial views of the first patient demonstrating a widened high forehead, narrowed nasal bridge, and malar hypoplasia. The cushingoid features are secondary to the chronic use of systemic steroids. A mild degree of asymmetrical axial proptosis is present.

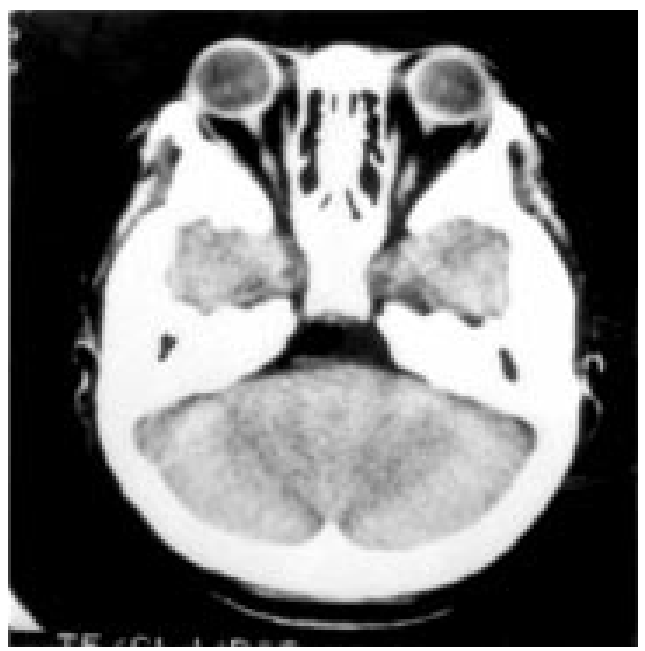

Figure 2 An axial view of a CT scan of the skull demonstrating thickened osteosclerotic bone consistent with progressive diaphyseal dysplasia.

Computed tomographic (CT) scanning of the brain and orbits demonstrated thickening of the skull consistent with PDD but with no associated stenosis of the optic canals (Fig 2). No dilatation of the ventricular system was noted. An intravenous digital subtraction angiogram showed no evidence of sagittal sinus thrombosis. At lumbar puncture, the opening pressure was elevated at $32 \mathrm{~cm}$ of water. Analysis of the cerebrospinal fluid (CSF) revealed no abnormalities.

A diagnosis of papilloedema was made but, as the patient had normal visual function, no treatment was given. Two months later, repeat kinetic perimetry demonstrated minimal bilateral peripheral visual field constriction with enlargement of the blind spots. The appearance of the optic discs was consistent with chronic papilloedema (Fig 3) and the patient was commenced on acetazolamide $250 \mathrm{mg}$ four times daily. The disc swelling improved, and the dose was reduced to $250 \mathrm{mg}$ twice over the subsequent 15 months.

In April 1991 the headaches returned and were accompanied by episodes of visual obscurations. The optic discs were more swollen; the visual fields were more constricted; and the dose of acetazolamide was increased to 250 mg three times daily with good effect. The serum alkaline phosphatase had risen concomitantly to 978 units/litre (normal value $<260 \mathrm{U} / \mathrm{l}$ ), suggesting that the clinical deterioration was related to an exacerbation of the bony dysplasia.

In June 1991 the patient complained of increasing deafness. Neuro-otological investigations demonstrated bilateral sensorineural hearing loss. Brainstem evoked potentials were abnormal, being absent on the left and of low amplitude and delayed on the right. A CT scan of the petrous temporal bones revealed bilaterally stenosed internal auditory canals and the patient therefore underwent decompression of the left vestibulocochlear nerve. At the last ophthalmic review in March 1997 visual acuity was $6 / 6$ bilaterally, visual fields were full and there was no optic disc swelling, acetazolamide having been discontinued 6 months previously.

CASE 2

This 39 year old male was diagnosed as having PDD in 1963 at the age of 8 on the basis of chronic bone pain, anaemia, and characteristic radiological changes affecting the limbs (Fig 4) and skull.

Between 1980 and 1990, he suffered recurrent episodes of papilloedema which were not associated with a deterioration in visual function and which were treated with lumbar puncture. 


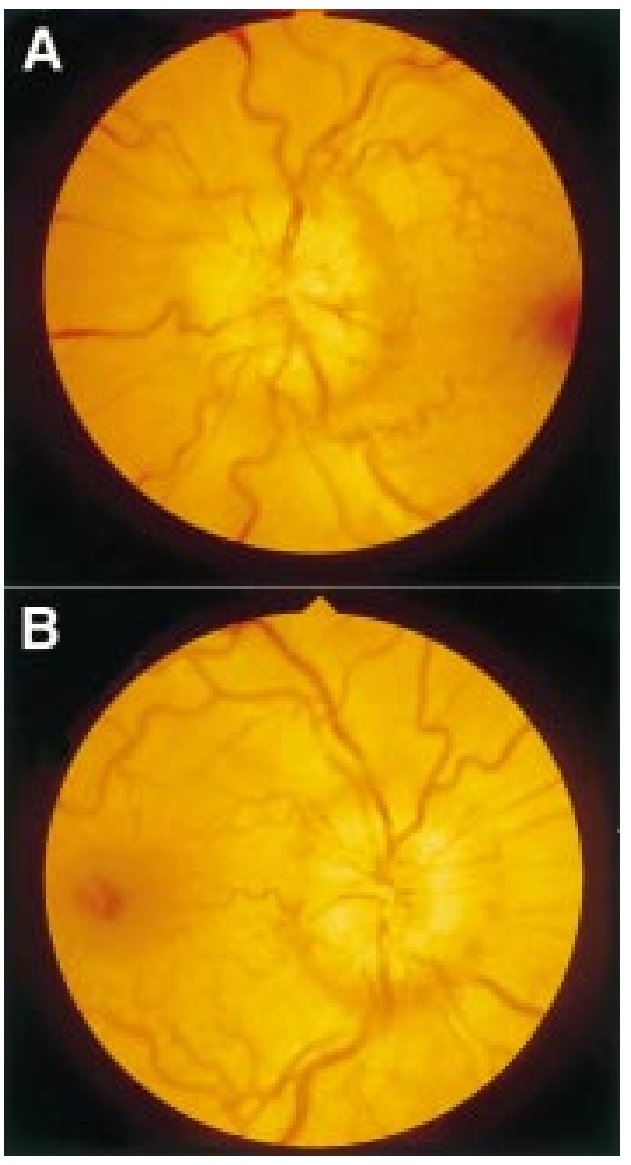

Figure 3 (A) and (B) Marked symmetrical disc oedema with hard exudates apparent within the superficial layers of the disc; the optic cup has been obliterated. The appearances are consistent with chronic papilloedema.

Acetazolamide therapy had been withdrawn because of a drug induced rash and on at least one occasion the papilloedema spontaneously resolved. Systemic steroid therapy had also been withdrawn following a steroid induced osteoporotic stress fracture affecting the lumbar vertebrae in 1977.

He was referred to the Wilmer Eye Institute in Baltimore in June 1993 after the acute onset of blurring in the inferior aspect of the left visual field accompanied by visual obscurations in that eye.

On examination, the patient had the typical facial appearance associated with PDD (Fig 5). $\mathrm{He}$ also had fixed flexion deformities of the arms and legs and enlargement of the long bones. Visual acuity was $6 / 5$ and N5 bilaterally, colour vision was normal, and no relative afferent pupillary defect was present. The visual field of the right eye showed enlargement of the blind spot with a supratemporal scotoma present. The left blind spot was sufficiently enlarged to encroach on fixation and was associated with a dense inferior arcuate scotoma. Both optic discs were swollen. A small peripapillary haemorrhage and an opticociliary shunt were present in the right ocular fundus; an extensive circumferential peripapillary subretinal haemorrhage was present in the left fundus (Fig 6).

There was no evidence of stenosis of the optic canals or sagittal sinus thrombosis with

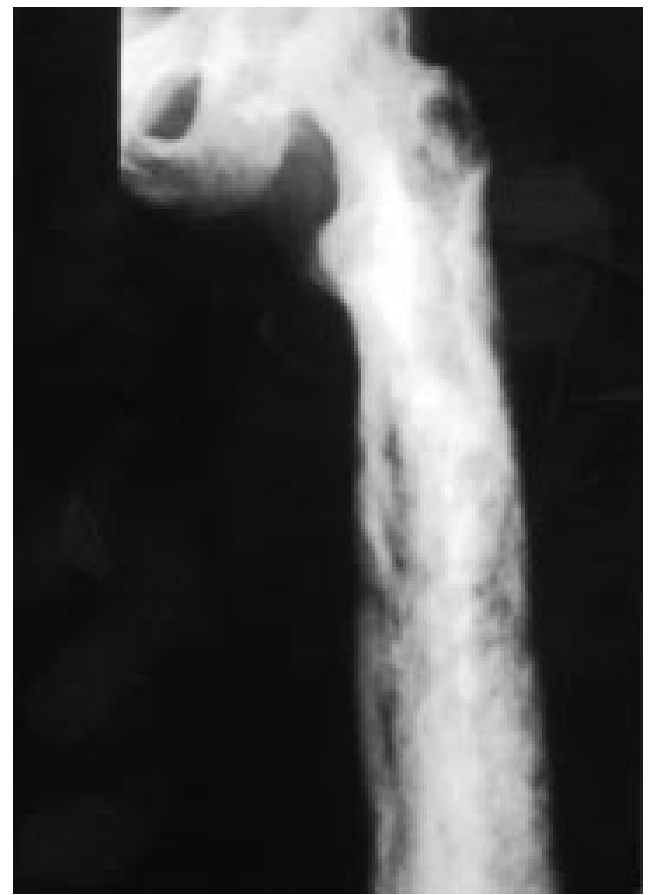

Figure 4 Radiograph of the left femur showing mottled areas of rarefaction of the bone with diaphyseal widening, thickened cortices, and narrowing of the medullary cavity, all of which are characteristic of progressive diaphyseal dysplasia.

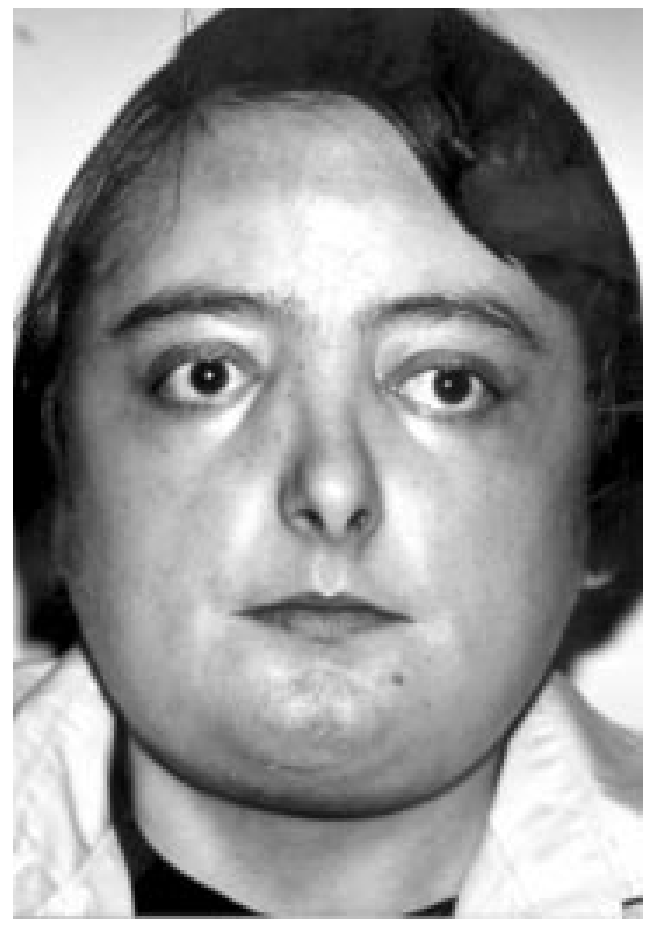

Figure 5 Photograph of the second patient demonstrating the typical facial characteristics observed in patients with progressive diaphyseal dysplasia.

CT scanning or magnetic resonance imaging (MRI). Orbital ultrasonography demonstrated enlargement of the optic nerves with a positive 30 degree test indicative of increased subarachnoid fluid. CT scanning and ultrasonography failed to demonstrate the presence of optic nerve head drusen. At lumbar puncture the opening pressure was $31 \mathrm{~cm}$ of water. Analysis 

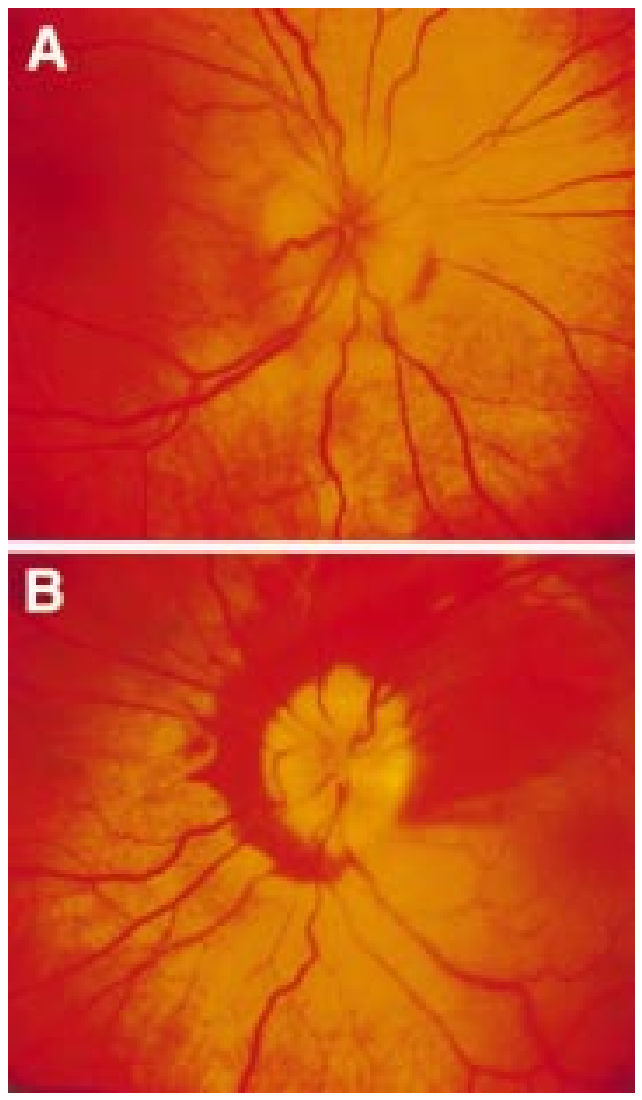

Figure 6 (A) and (B) Both optic discs appear swollen. The prominent opticociliary shunt observed in the right ocular fundus was not present in photographs taken in 1986. There is an extensive circumferential peripapillary subretinal haemorrhage present in the left ocular fundus.

of the CSF gave normal results. Serum alkaline phosphatase was raised at $445 \mathrm{U} / 1$.

Both optic discs became more swollen over the next 6 weeks, but the peripapillary haemorrhages and visual field defects improved.

At last review in December 1996, the optic disc swelling had spontaneously resolved (Fig 7) and spontaneous venous pulsations were present. Visual acuities were $6 / 5$ right, $6 / 6$ left, visual fields were full, with only minimal enlargement of the blind spots.

CASE 3

This 56 year old female first presented in 1946 at age 5 years with a painful gait, secondary muscle atrophy, and radiological changes of the skull and long bones which were typical of PDD. At age 16 years, she presented with bilateral progressive proptosis and optic disc swelling. The optic foramina were of normal size on skull radiographs and no abnormalities were noted on ventriculography; however, the level of the intracranial pressure (ICP) was not documented. Alkaline phosphatase was elevated at $475 \mathrm{U} / 1$. A cranial vault expansion combined with bilateral orbital decompressions was performed with resolution of the proptosis and disc oedema.

The patient developed slowly progressive, profound bilateral sensorineural deafness from the age of 22. Tomography of the petrous temporal bones demonstrated severe stenosis of both internal auditory meati.
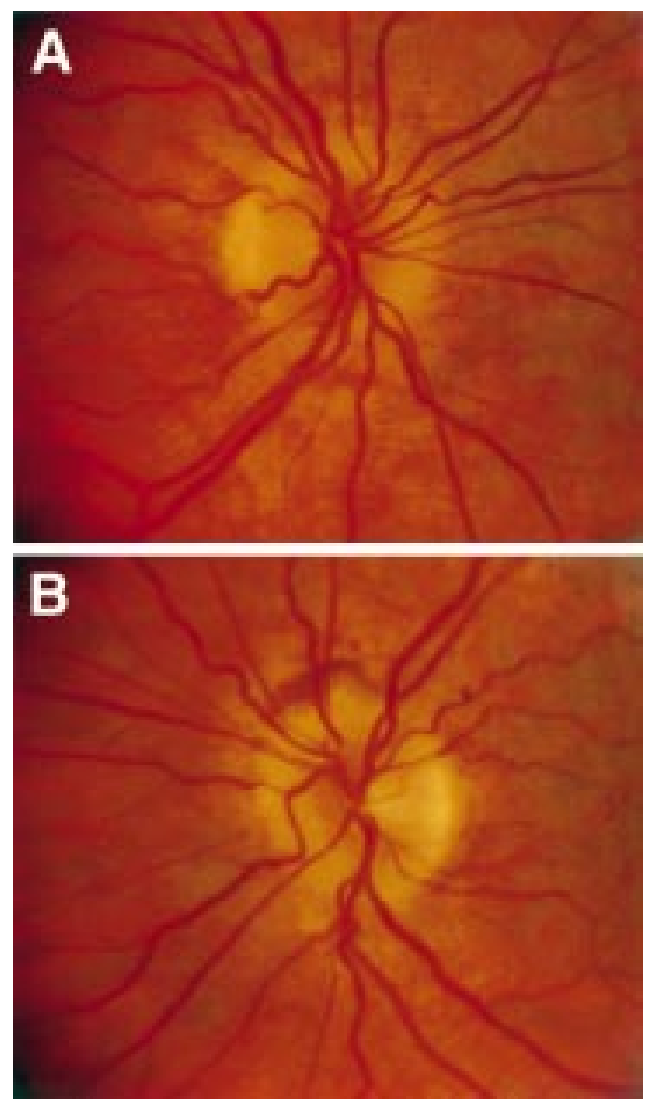

Figure 7 (A) and (B) Spontaneous resolution of the optic disc swelling with minimal left sided superior peripapillary pigment epithelial change.

The patient underwent unsuccessful trials of medical therapy, firstly with calcitonin in 1974 and subsequently with the biphosphonate etidronate in 1982. Her bone pain remains well controlled on prednisolone $10 \mathrm{mg}$ daily. In 1983 the patient developed a left sided corneal abscess secondary to upper lid entropion which required surgical correction.

She subsequently presented to the Institute of Neurological Sciences in Glasgow in January 1988 with bilateral visual blurring and ocular irritation.

On examination, the typical facial features associated with PDD were modified by her previous craniofacial surgery and the effects of long term systemic steroid therapy (Fig 8). Visual acuities were 6/18 right and 6/24 left. The visual field of the right eye was full; the visual field of the left eye was constricted nasally. Minimal lower motor neuron facial nerve weakness with intact Bell's phenomenon was present bilaterally. There was evidence of chronic exposure keratopathy with superficial punctate erosions and inferior corneal neovascularisation, more marked on the left side. Both optic discs appeared minimally pale but within normal limits. Diagnostic syringing confirmed the presence of bilateral nasolacrimal duct blockage which was probably secondary to a combination of her mid facial hypoplasia and an osteosclerotic stenosis of the nasolacrimal duct.

Skull radiography demonstrated diffuse osteosclerotic changes in addition to the tanta- 


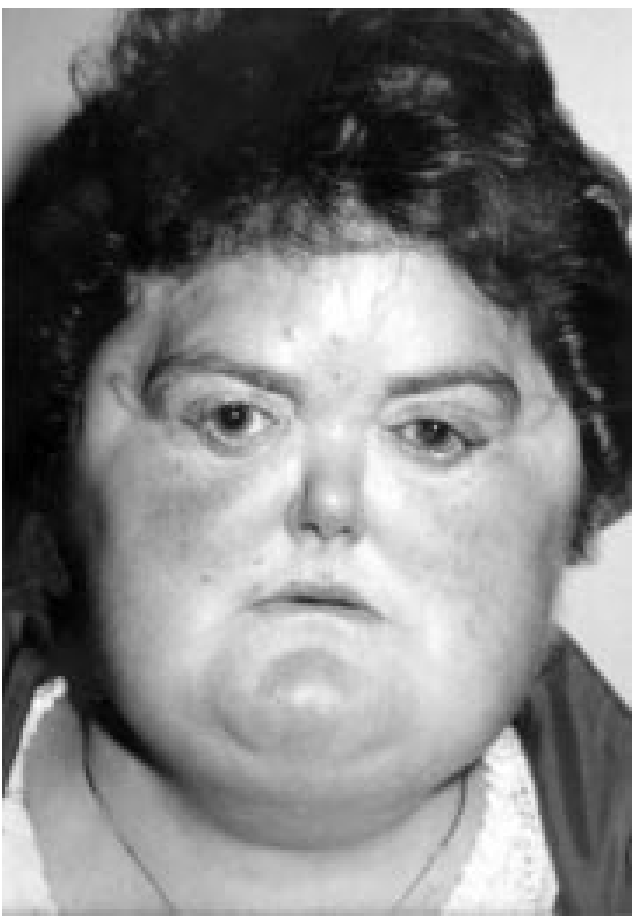

Figure 8 Despite the previous craniofacial surgery, the photograph of the third patient demonstrates similar facial characteristics to those seen in the previous two patients. Lateral tarsorrhaphies have been performed.

lum plates which had been inserted as part of the previous cranial expansion (Fig 9). The lesser wings of the sphenoid bone appear unusually prominent as both the vertical and orbital portions of the frontal bone have been removed as part of the orbital decompression. An axial CT scan demonstrated diffuse thickening of the bones of the vault and base of the skull, bone defects were noted in the roof of both orbits (Fig 10).

Bilateral lateral tarsorrhaphies were performed, and ocular lubricants were continued. Despite initial improvement, over a 2 year period the patient developed recurrent left corneal ulceration with corneal decompensation and with formation of a descemetocele. At the last ophthalmic consultation in May 1997 the visual acuities were $6 / 9$ and counting fingers with minimal peripheral constriction of the visual fields.

\section{Results}

All three patients had bilateral optic disc swelling with no radiological evidence of either compressive optic neuropathy or thrombosis of the intracranial venous sinuses. The aetiology of the disc swelling was proved to be papilloedema in the first two cases and was probably the dominant cause in the third case. The papilloedema resolved spontaneously in the second case and after treatment with acetazolamide in the first case.

\section{Discussion}

AETIOLOGY OF RAISED INTRACRANIAL PRESSURE The aetiology of raised ICP in patients with PDD is probably multifactorial. Skull base involvement with stenosis of the jugular foramina has been demonstrated on MR

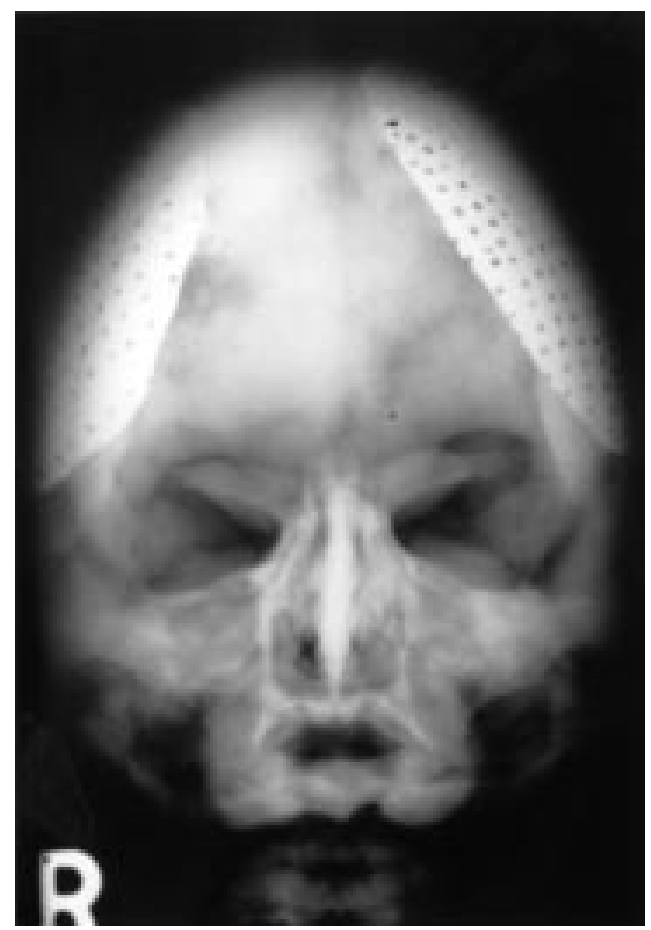

Figure 9 Plain skull radiograph demonstrating diffuse hyperostosis and sclerosis affecting the frontal and parietal bones in addition to the tantalum plates which were inserted as part of the cranial vault expansion.

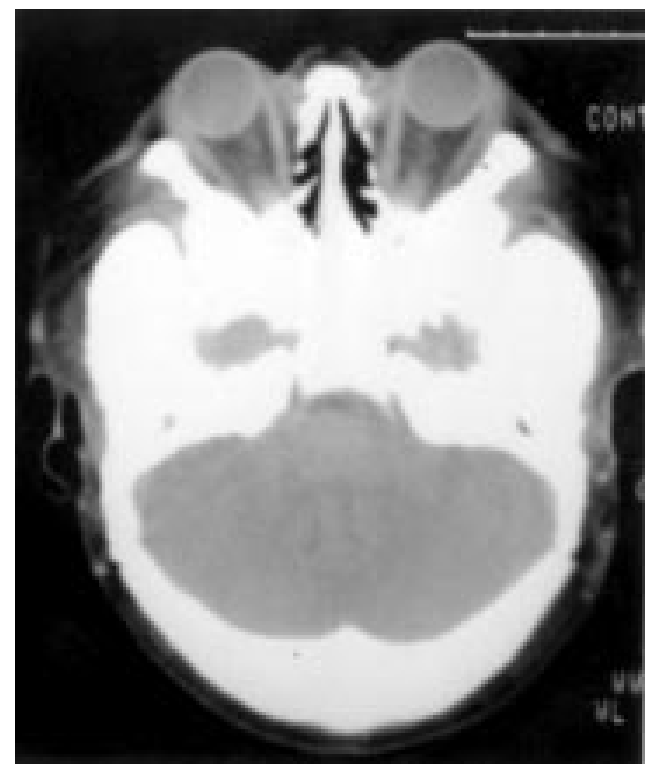

Figure 10 An axial view of a CT head scan demonstrating diffuse thickening of the bones of the vault and base of the skull. Bone defects are noted in the roof of both orbits which correspond to the site of the previous orbital decompression

scanning, ${ }^{13}$ and angiography has demonstrated distortion of the intracranial vessels. ${ }^{14}$ Both abnormalities are considered to be indirect evidence of impairment of venous drainage. Neuroimaging failed to demonstrate evidence of sagittal or lateral sinus thrombosis in our patients. Encroachment of the cranial contents by progressive thickening of the calvarial bone may contribute to the raised ICP; however, we observed no effacement of the ventricles on CT or MRI. 
Most reports of benign intracranial hypertension $(\mathrm{BIH})$ occurring in association with systemic corticosteroid treatment have concerned young children, although there have been reported cases involving adults. The onset of BIH usually occurs on withdrawal of corticosteroids after prolonged treatment ${ }^{15}$; however, several reports exist of $\mathrm{BIH}$ occurring during their use. ${ }^{16}$ The dose of prednisolone taken by our first patient had remained stable before her presentation with papilloedema. Our second patient experienced relapses of his papilloedema many years after stopping corticosteroid treatment and our third patient was not taking corticosteroids when she developed disc swelling. This suggests that in the two patients in whom the ICP was measured, the episodes of raised ICP were unrelated to the use or withdrawal of systemic corticosteroids.

The spontaneous remissions of the elevated ICP in the second patient are difficult to explain but probably reflect autoregulatory mechanisms. These affect the balance between CSF production and absorption which can overcome the compressive effects associated with bony enlargement of the skull. In at least two of our cases, the episodes of raised ICP seem to have been temporally related to elevations of serum alkaline phosphatase, indicating an exacerbation of the bony dysplasia and suggesting a causal relation.

AETIOLOGY OF VISUAL FAILURE

In all previous reported cases of visual failure associated with PDD, ${ }^{10} 11{ }^{17}{ }^{18}$ with possibly one exception, ${ }^{14}$ the aetiology of the visual loss has been assumed to have occurred solely as a result of a compressive optic neuropathy. PDD is part of the spectrum of primary bone disorders which include craniometaphyseal dysplasia, fibrous dysplasia, osteopetrosis, and aneurysmal bone cysts, all of which have been reported to cause progressive stenosis of the optic canals. ${ }^{19-22}$ Although raised ICP has been described in patients with PDD in whom visual loss has occurred, the elevation of ICP in those patients has been thought to be either non-contributory ${ }^{17}$ or only partially responsible ${ }^{14}$ for the accompanying visual loss.

Yen et $a l^{17}$ reported a patient who underwent a successful combined bilateral orbital and optic canal decompression and who was incidentally found to have elevated ICP $(23 \mathrm{~cm}$ of water preoperatively and $28 \mathrm{~cm}$ postoperatively). As with other disorders of bone metabolism, papilloedema can either cause visual loss or coexist with a compressive optic neuropathy and appear to cause visual loss. Van Dalsem et $a l^{14}$ reported a case in which optic nerve compression and papilloedema coexisted; both were thought to contribute to the visual impairment.

The occurrence of peripheral and paracentral visual field constriction with preservation of colour vision and acuity suggests that the dominant cause of the acute visual loss observed in our patients is secondary to papilloedema rather than a compressive optic neuropathy which tends to reduce central acuity and colour vision early.
Both the first and second of our cases had papilloedema with no radiological or clinical evidence of a compressive optic neuropathy. The disc swelling resolved spontaneously in the second case and following treatment with acetazolamide in the first case.

Our third patient was thought to have significant bony encroachment of the cranial cavity, with no radiological evidence of optic canal stenosis. As the disc swelling resolved after a combined cranial vault expansion and orbital decompression procedure, it would seem likely that the visual failure she experienced in childhood was at least in part secondary to raised ICP.

\section{TREATMENT OPTIONS}

Persistently raised ICP occurring in association with progressive deterioration in visual function is an indication for aggressive treatment; however, both CSF shunting procedures and optic nerve sheath fenestration can fail over time. Based upon the long term success obtained in our last patient and that reported following subtemporal decompression, ${ }^{14}{ }^{23}$ we believe that some type of cranial vault expansion procedure, possibly combined with an orbital decompression, would be worthy of consideration in these patients.

Surgical intervention in PDD is not straightforward. The results of combined orbital and optic canalicular decompression have been reported in two patients. However, tomography of the optic canals and orbital roofs performed 8 months to 1 year postoperatively demonstrated complete reconstitution of the surgically resected bone. ${ }^{10}{ }^{17}$ Interestingly, the skull radiographs and CT scans of our third patient demonstrated a lack of bony regrowth following the removal of both the vertical and orbital portions of the frontal bone as part of the orbital decompression performed 31 years earlier.

Regardless of whether or not surgical intervention is indicated in patients with PDD, the definitive treatment of such patients is likely to rest in the long term control of the abnormal formation of bone. The administration of systemic corticosteroids has been shown to decrease the abnormally high diaphyseal uptake of $99 \mathrm{~m}$-technetium methylene diphosphonate, which is thought to represent focal osteoblastic overactivity. ${ }^{24}$ Steroids are also effective in alleviating limb pain and muscle weakness and improve the quality of life although not necessarily altering the course of the disease. ${ }^{25}$ Biphosphonates such as disodium etidronate are adsorbed onto hydroxyapatite crystals reducing their rate of growth and dissolution and retarding the mineralisation of osteoid and therefore, the rate of bone turnover. Our third patient failed to benefit symptomatically, biochemically, or radiologically from a 4 month therapeutic trial of etidronate. The only other report of the use of this drug in PDD is limited to one case report in which the elevation in alkaline phosphatase activity was reduced but not normalised after 7 weeks of treatment. ${ }^{26}$ Calcitonin has been used to treat the bone pain and neurological 
complications of Paget's disease, but we are unaware of any reports of its use in patients with PDD. Neither our first nor our third patient improved after short therapeutic trials of calcitonin.

In summary, the visual loss documented in at least two of the three patients described above appears to be solely attributable to raised ICP. Normalisation of the ICP has led to an improvement and stabilisation of the visual function in all three patients.

We thank the three patients for permission to report their cases.

1 Camurati $M$. Di un raro caso osteite simmetrica ereditaria degli arti inferiori. Chir Organi Mov 1922;6:662.

2 Engelmann G. Ein fall von osteopathia hyperostotica (sclerotisans) multiplex infantilis. Fortschir Geb Roentgen 1929; 39:1101

3 Resnick D, Niwayama G. In: Diagnosis of bone and joint disorders. 2nd ed. Vol 5. Philadelphia: Saunders, 1988:3483-4

4 Fallon MD, Whyte MP, Murphy WA. Progressive diaphyseal dysplasia (Engelmann's disease): report of a sporadic case of the mild form. F Bone Foint Surg 1980;62A:465.

5 Hundley JD, Wilson FC. Progressive diaphyseal dysplasia. A review of the literature and a report of seven cases in one family. F Bone foint Surg 1973;55A:461.

6 Sparkes RS, Graham CB. Camurati-Engelmann disease. Genetics and clinical manifestations with a review of the literature. 7 Med Genet 1972:9:73-85.

7 Naveh Y, Kaftori JK, Alon U, et al. Progressive diaphyseal dysplasia: genetics and clinical and radiological manifestations. Pediatrics 1984;74:399-405.

8 Kaftori JK, Kleinhaus MD, Naveh YH. Progressive diaphyseal dysplasia (Camurati-Engelmann): radiographic seal dysplasia (Camurati-Engelmann): radiographic

9 Wirth CR, Kay J, Bourke R. Diaphyseal dysplasia (Engelmann's syndrome). A case report demonstrating a deficiency in cortical haversian system formation. Clin Orthop 1982;171:186-95.

10 Krohel GB, Wirth CR. Engelmann's disease. Am $\mathcal{F}$ Ophthalmol 1977;84:520-5.
11 Morse PH, Walsh FB, McCormick JR. Ocular findings in hereditary diaphyseal dysplasia. Am F Ophthalmol 1969;68: 00-4.

12 Brodrick JD. Luxation of the globe in Engelmann's disease. Am f Ophthalmol 1977;83:870-3.

13 Applegate LJ, Applegate GR, Kemp SS. MR of multiple cranial neuropathies in a patient with CamuratiEngelmann disease: case report. Am f Neurorad 1991;12: 557-9.

14 Van Dalsem VF, Genant HK, Newton TH. Progressive diaphyseal dysplasia a report of a case with 34 years of progressive disease. F Bone foint Surg 1979;61A:596-8.

15 Neville BGR, Wilson J. Benign intracranial hypertension following corticosteroid withdrawal in childhood. BMF 1970;3:554-6.

16 Newton M, Cooper BT. Benign intracranial hypertension during prednisolone treatment for inflammatory bowel disease. Gut 1994;35:423-5.

17 Yen JK, Bourke RS, Popp AJ, et al. Camurati-Engelmann disease (progressive hereditary diaphyseal dysplasia). $\mathcal{F}$ Neurosurg 1978;48:138-42.

18 Walsh FB, Hoyt WF. Clinical neuro-ophthalmology. 3rd ed. Vol 2. Baltimore: Williams and Wilkins, 1969:1068-9.

19 Puliafito CA, Wray SH, Murray JE, et al. Optic atrophy and visual loss in craniometaphyseal dysplasia. Am $\mathcal{f}$ Ophthalmol 1981;92:696-701.

20 Calderon M, Brady HR. Fibrous dysplasia of bone with bilateral optic foramnia involvement. Am $\mathcal{f}$ Ophthalmol 1969;68:513-5.

21 Aasved $\mathrm{H}$. Osteopetrosis from the ophthalmological point of view. Acta Ophthalmol 1970;48:771-8.

22 Yee RD, Cogan DG, Thorp TR, et al. Optic nerve compression due to aneurysmal bone cyst. Arch Ophthalmol 1977;95:2176-9.

23 Mottram ME, Hill HA. Diaphyseal dysplasia: report of a case. Am f Roentg 1965;95:162-7.

24 Verbruggen LA, Bossuyt A, Schreuer R, et al. Clinical and scintigraphic evaluation of corticosteroid treatment in a case of progressive diaphyseal dysplasia. F Rheumatol 1985; 12:809-13.

25 Naveh Y, Alon U, Kaftori JK, et al. Progressive diaphyseal dysplasia: evaluation of corticosteroid therapy. Pediatrics 1984;75:321-3.

26 Smith R, Walton RJ, Corner BD, et al. Clinical and biochemical studies in Engelmann's disease (progressive diaphyseal dysplasia). $Q \mathcal{F}$ Med 1977;182:273-93. 